\title{
Combinatorial properties of stable spin curves
}

\author{
Lucia Caporaso and Cinzia Casagrande
}

\author{
Lucia Caporaso \\ Dipartimento di Matematica, Università di Roma Tre \\ Largo S. L. Murialdo, 1 \\ 00146 Roma - ITALY \\ caporaso@mat.uniroma3.it \\ Cinzia Casagrande \\ Dipartimento di Matematica, Università di Roma "La Sapienza" \\ Piazzale Aldo Moro, 2 \\ 00185 Roma - ITALY \\ ccasagra@mat.uniroma1.it
}

Dedicated to Professor Steven Kleiman, for his birthday

\section{Stable spin curves and their moduli}

\subsection{Summary}

The first part of this paper describes the moduli space of stable spin curves (constructed in [Cor89]), explaining how its geometry is governed by the combinatorics of stable curves. In this context, the standard graph theoretic framework (where to every stable curve one associates its "dual graph") is not just a book-keeping device: in Section 2, some purely combinatorial results are proved (Theorems 11 and 13), having moduli theoretic applications. More precisely, certain strata of the moduli space of stable curves are characterized by a (finite) set of integers that measures the non-reducedness of the scheme of spin curves, and which is definable in purely graph-theoretical terms (Definition 7).

\subsection{The basic functors and their compactifications}

A smooth spin curve is a pair $(X, L)$ where $X$ is a smooth, connected, projective curve of genus $g$ and $L$ a theta-characteristic of $X$, that is, a line bundle such that $L^{\otimes 2}=K_{X}$.

\footnotetext{
${ }^{1} 2000$ Mathematics Subject Classification: 14H10, 05C75
} 
Smooth spin curves naturally define a coarsely representable functor: to any family $\mathcal{X} \longrightarrow B$ of smooth curves (i.e. the fiber $X_{b}$ over every point $b \in B$ is a smooth curve) it associates the relative spin curve

$$
\mathcal{S}_{\mathcal{X} / B} \longrightarrow B
$$

whose fiber over $b \in B$ is the set of $2^{2 g}$ smooth spin curves supported on $X_{b}$ (i.e. the set of theta-characteristics of $X_{b}$ ). To complete the picture, consider the Picard functor: denote by

$$
\operatorname{Pic}_{\mathcal{X} / B}^{d} \longrightarrow B
$$

the relative, degree $d$, Picard variety, whose fiber over $b \in B$ is the variety parametrizing line bundles of degree $d$ on $X_{b}$. We have a commutative diagram:

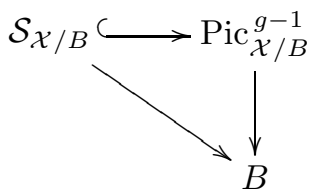

Because of the coarse representability of all the functors involved, we get a global picture

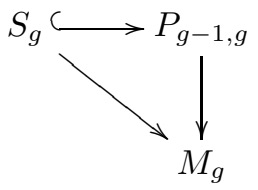

where $M_{g}$ is the moduli space of smooth curves of genus $g \geq 2, S_{g}$ the moduli space of smooth spin curves and $P_{d, g}$ the universal Picard variety of degree $d$, parametrizing isomorphism classes of degree $d$ line bundles over smooth curves of genus $g$ (the diagram, of course, represents only the case $d=g-1$ ).

The word "space" here means either algebraic stack or algebraic scheme; the warning is the usual one: in the category of schemes, the moduli properties of the above diagram fail for objects with non-trivial automorphisms.

In order to compactify $M_{g}$, P. Deligne and D. Mumford introduced stable curves (in [DM69).

Definition 1. A stable curve is a reduced, connected curve having only ordinary double points as singularities and ample dualizing sheaf. This last condition is equivalent to the fact that every smooth rational component of the curve contains at least 3 nodes.

By weakening this last requirement, asking that the nodes contained in any smooth rational component be at least 2 , one gets the definition of a semistable curve. Thus a semistable curve $X$ is a nodal curve that fails from being stable if it contains some smooth, rational component $E$ such that $\#(\overline{X \backslash E} \cap X)=2$. Such an $E$ will be called exceptional.

A quasistable curve is a semistable curve such that two exceptional components never meet each other.

The stable model of a semistable curve is the uniquely defined stable curve obtained by contracting every exceptional component to a point. 
Stable curves of (arithmetic) genus $g \geq 2$ have a moduli space, denoted by $\overline{M_{g}}$, which is projective and contains $M_{g}$ as a dense open subspace.

Having compactified $M_{g}$ in this fashion, the problem arises on how to compactify $S_{g}$ and $P_{d, g}$ accordingly.

A solution for $S_{g}$ was given by M. Cornalba in Cor89, consistently with the DeligneMumford construction. He defined stable spin curves:

Definition 2. A stable spin curve is a pair $(Y, L)$ where $Y$ is a quasistable curve and $L$ a line bundle on $Y$ with the following properties. Denote by $E$ any exceptional component of $Y$ and by $Z:=\overline{Y \backslash \cup E}$ the closure of the complement of all exceptional components; then the restriction of $L$ to every exceptional component $E$ is $\mathcal{O}_{E}(1)$, and the restriction to what remains satisfies

$$
L_{\mid Z}^{\otimes 2} \cong \omega_{Z}
$$

Notice that the degree of $L$ is $g-1$.

Stable spin curves are shown in Cor89 to have a projective moduli space $\overline{S_{g}}$, with a natural, finite morphism of degree $2^{2 g}$ onto $\overline{M_{g}}$

$$
\pi: \overline{S_{g}} \longrightarrow \overline{M_{g}} \text {. }
$$

The fiber of $\pi$ over a stable curve $X \in \overline{M_{g}}$ is a zero-dimensional scheme parametrizing stable spin curves $(Y, L)$ such that the stable model of $Y$ is $X$.

With diagram (1) in mind, the question remains on how to compactify $P_{d, g}$; the problem of completing the Picard functor is rich with many aspects, which will not be described here, and has been (and still is) the object of interest for a long time. For solutions and methods to approach it, that differ from what will be presented in this paper, we refer to the recent articles [AK90] and EGK02] of A. Altman, S. Kleiman, E. Esteves, M. Gagné, and to the references therein.

In this paper, we are interested in stable curves (thus, we only allow nodal singularities); furthermore, we consider completions over $\overline{M_{g}}$ of the Spin functor and, marginally, of the Picard functor, that use polarized quasistable curves as boundary points. The first to be completed was $S_{g}$, by the above described space $\overline{S_{g}}$.

A compactification, $\overline{P_{d, g}}$, of $P_{d, g}$ over $\overline{M_{g}}$, was later constructed in Cap94; briefly said, $\overline{P_{d, g}}$ parametrizes pairs $(Y, M)$ where $Y$ is a quasistable curve of genus $g, M$ is a line bundle of degree $d$ on $Y$, having degree 1 on all exceptional components and satisfying other "degree constraints" (which we shall not explain here).

The evident similarity between the boundary points of $\overline{S_{g}}$ and $\overline{P_{d, g}}$ seemed a bit striking, since the two constructions were independent and used different techniques. Only recently, C. Fontanari ( a subscheme of $\overline{P_{g-1, g}}$, so that one simultaneously compactifies all objects in (1):

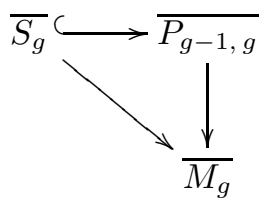


The above diagram, where the standard functoriality properties are satisfied, clarifies and highlights the "naturality" of the boundary objects: stable curves, spin stable curves, suitably polarized quasistable curves. We shall keep it in mind throughout the paper.

\subsection{Stable spin curves}

We start by recalling some facts about stable spin curves, referring to Cor89 for detailed proofs. Fix a stable curve $X$ and let $(Y, L)$ be a stable spin curve such that the stable model of $Y$ is $X$; the quasistable curve $Y$ will be called the support of the spin curve. We shall denote by $S_{X}$ the moduli space of stable spin curves whose support has $X$ as stable model . Thus, if $X$ has trivial automorphism group, $S_{X}$ is the the (scheme-theoretic) fiber of $\pi: \overline{S_{g}} \longrightarrow \overline{M_{g}}$ over $X$.

Let $\nu: X^{\nu} \longrightarrow X$ be the normalization map, $C \subset X$ an irreducible component and $C^{\nu}$ the corresponding component in $X^{\nu}$. Let $\Delta \subset X_{\text {sing }}$ be a set of nodes of $X$, set $\Delta_{C}:=\Delta \cap C$ and denote by $D_{C} \subset C^{\nu}$ the preimage of $\Delta_{C}$, i.e. $D_{C}:=\nu^{-1}\left(\Delta_{C}\right)$. Thus $D_{C}$ is an effective, reduced divisor of $C^{\nu}$.

Definition 3. We say that $\Delta$ is even if, for every irreducible component $C$ of $X$, $\operatorname{deg} D_{C}$ is even.

For example, the empty set is even.

Notice that, equivalently, $\Delta$ is even if, denoting by $Z$ the partial normalization of $X$ at all nodes that are not in $\Delta$, the dualizing sheaf $\omega_{Z}$ has even degree on every irreducible component of $Z$.

Consider now the set of all quasistable curves having $X$ as stable model; this set is obviously finite and in bijective correspondence with the set of subsets of nodes of $X$. More precisely, let $Y$ be a quasistable curve and let $\sigma: Y \longrightarrow X$ be the natural morphism contracting all the exceptional components of $Y$. Denote by $\Delta_{Y} \subset X_{\text {sing }}$ the set of nodes corresponding to the nodes of $Y$ that are not contained in an exceptional component:

$$
\Delta_{Y}:=\sigma(Y \backslash \cup E)_{\text {sing }} .
$$

Clearly $Y$ uniquely determines $\Delta_{Y}$ and, conversely, for every $\Delta \subset X_{\text {sing }}$ there exists a unique quasistable curve $Y$ such that $\Delta_{Y}=\Delta$.

A basic consequence of Cornalba's construction is the following

Fact. A quasistable curve $Y$ is the support of a spin curve if and only if $\Delta_{Y}$ is even.

Having characterized all quasistable curves appearing as supports of spin curves, we fix one, $Y$, and describe all line bundles $L \in \operatorname{Pic} Y$ such that $(Y, L) \in S_{X}$; as always, $\nu: Y^{\nu} \longrightarrow Y$ denotes the normalization.

Denote $L^{\nu}:=\nu^{*} L$, so that $L^{\nu}$ is the datum of a line bundle on each irreducible component of $Y^{\nu}$. By Definition 2, we have

$$
L_{\mid E}^{\nu}=\mathcal{O}_{E}(1)
$$


and, for every non-exceptional component $C$ of $Y$

$$
\left(L_{\mid C^{\nu}}^{\nu}\right)^{\otimes 2}=K_{C^{\nu}} \otimes \mathcal{O}\left(D_{C}\right) .
$$

This last formula follows from Definition 2: recall that, denoting $Z:=\overline{Y \backslash \cup E}, L$ satisfies

$$
L_{\mid Z}^{\otimes 2} \cong \omega_{Z}
$$

Fix now $L^{\nu}$ as above; the set of all line bundles $L$ on $Z$ that satisfy (3), and pull back to $L^{\nu}$ (restricted to $Z^{\nu}$ ), is found by looking at the exact sequence of algebraic groups:

$$
1 \longrightarrow\left(\mathbb{C}^{*}\right)^{b_{1}\left(\Gamma_{Z}\right)} \longrightarrow \operatorname{Pic} Z \stackrel{\nu^{*}}{\longrightarrow} \operatorname{Pic} Z^{\nu} \longrightarrow 0
$$

where $\Gamma_{Z}$ is the dual graph of $Z$ (whose definition we recall below), and $b_{1}\left(\Gamma_{Z}\right)$ its first Betti number.

Definition 4. Let $Z$ be a reduced nodal curve. The dual graph of $Z$, denoted by $\Gamma_{Z}$, is the graph whose vertices are the irreducible components of $Z$ and whose edges are the nodes of $Z$.

The above sequence says that there are $\left(\mathbb{C}^{*}\right)^{b_{1}\left(\Gamma_{Z}\right)}$ line bundles on $Z$, all pulling back

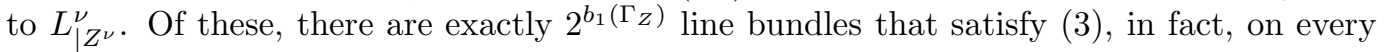
node of $Z$, there are exactly two gluings compatible with (3).

The gluing data on the remaining nodes of $Y$, lying on some exceptional component, do not give different isomorphism classes of spin curves. More precisely, let $N$ be any node of $Y$ that lies on an exceptional component; different gluings of $L^{\nu}$ over $N$ give the same point of $S_{X}$, but determine the scheme structure of $S_{X}$ at such a point, being responsible for the non-reducedness of $S_{X}$.

Set

$$
b=b_{1}\left(\Gamma_{X}\right)=b_{1}\left(\Gamma_{Y}\right)
$$

and notice that

$$
b=\# Y_{\text {sing }}-\#\{\text { irreducible components of } Y\}+1=g-p
$$

where

$$
p:=\sum_{C} p_{g}(C),
$$

$p_{g}(C)$ is the geometric genus of $C$ and the the sum is extended to all irreducible components $C$ of $X$ (or of $Y$ ).

By what we said, $S_{X}$ contains $2^{b_{1}\left(\Gamma_{z}\right)}$ distinct points $(Y, L)$ such that $\nu^{*} L=L^{\nu}$. At each of these points, the multiplicity of $S_{X}$ is computed in Cor89, Section 4 and in CS02] Section 2.2 ; it is equal to $2^{b-b_{1}\left(\Gamma_{z}\right)}$.

Let us recapitulate and check the above analysis by computing the length of $S_{X}$ (which must, of course, be equal to $2^{2 g}$ ). 
The number of choices for $Y$ is equal to the number of even subsets on nodes of $X$, which is equal to the number of cyclic subgraphs of $\Gamma_{X}$ (see the remark in Section 2.1), which is equal to $2^{b}$.

The number of choices of $L^{\nu} \in \operatorname{Pic} Y^{\nu}$ is $2^{2 p}$ (recall that $p=\sum p_{g}(C)$ ); in fact, for every irreducible component $C$ of $Y$, the number of choices for the restriction of $L^{\nu}$ to $C^{\nu}$ is equal to $2^{2 p_{g}(C)}$ (by formula (2)).

For each $L^{\nu}$ we have $2^{b_{1}\left(\Gamma_{Z}\right)}$ distinct points of $S_{X}$, corresponding to the different gluings over the nodes of $Z$ (by (印). All such points have multiplicity $2^{b-b_{1}\left(\Gamma_{Z}\right)}$. Summarizing:

$$
\text { length }\left(S_{X}\right)=2^{b} \cdot 2^{2 p} \cdot 2^{b_{1}\left(\Gamma_{Z}\right)} \cdot 2^{b-b_{1}\left(\Gamma_{Z}\right)}=2^{2 b+2 p}=2^{2 g} .
$$

We shall now make a small change of notation, to better highlight how the scheme structure of $S_{X}$ is governed by the combinatorics of $X$.

To a set $\Delta$ of nodes of $X$, one associates a subgraph of $\Gamma_{X}$ as follows: $\Delta$ is identified with a set of edges of $\Gamma_{X}$, which naturally generates a subgraph, which is the smallest subgraph of $\Gamma_{X}$ containing $\Delta$. For example, in the above set up, the graph associated to $\Delta_{Y}$ is identifiable to the dual graph of $Z, \Gamma_{Z}$. We will abuse notation and denote by the same symbol, $\Delta$, the set of nodes of the curve, the corresponding set of edges of the dual graph, and the subgraph generated by such edges. Therefore, in the previous set up

$$
b_{1}\left(\Gamma_{Z}\right)=b_{1}\left(\Delta_{Y}\right) .
$$

Having done that, we summarize the above analysis of the structure of $S_{X}$ :

Proposition 5 (Numerics of $S_{X}$ ). $S_{X}$ is a zero-dimensional scheme of length $2^{2 g}$. The number of its irreducible components is

$$
2^{2 p} \cdot\left(\sum_{\Delta \subset X_{\text {sing }}, \Delta \text { even }} 2^{b_{1}(\Delta)}\right)
$$

A component of $S_{X}$ parametrizing the stable spin curve $(Y, L)$ appears with multiplicity equal to $2^{b-b_{1}\left(\Delta_{Y}\right)}$.

\subsection{Examples}

Example 1 ( $S_{X}$ is reduced if and only if $X$ is of compact type). Let $X$ be a curve of compact type; then $b=0$ and the only even set of nodes of $X$ is $\emptyset$. Obviously, $b_{1}(\emptyset)=0$, therefore $S_{X}$ is reduced and all of its points parametrize pairs $(Y, L)$ such that $Y$ is the quasistable curve obtained by "blowing up" every node of $X$ and $L$ is the datum of a theta characteristic on every irreducible component of $X$.

Conversely, suppose that $S_{X}$ is reduced. Then, by the proposition, for every even subset $\Delta$ of nodes of $Y$ we must have

$$
b-b_{1}(\Delta)=0 .
$$

In particular $(\emptyset$ is even)

$$
b-b_{1}(\emptyset)=b=0
$$

therefore $X$ is of compact type. 
Example $2(b=1)$. If $b=1$ then $p=g-1$. Denote by $W$ a (possibly empty) disjoint union of a finite number of curves of compact type $W_{i}$ : $W=\cup W_{i}$, with $W_{i} \cap W_{j}=\emptyset$. There are two possibilities:

(a) $X=C \cup W$ with $W$ as above, $C$ irreducible with one node $N$, and $\# C \cap W_{i}=1$ for every $i$;

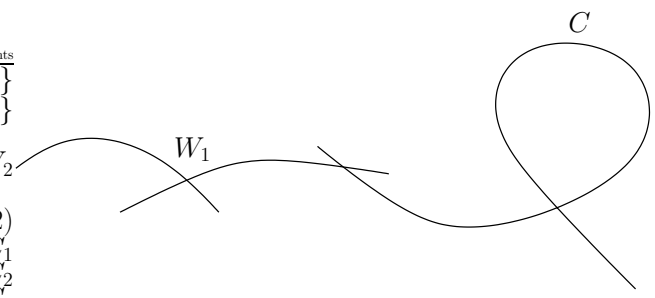

Case (1)

(b) $X=D \cup W$ such that $W$ is as above, $D$ is a "cycle" of smooth components, that is, $D=C_{1} \cup \ldots \cup C_{h}$ with $C_{i}$ smooth, $C_{i}$ intersects $C_{j}$ if and only if $i$ and $j$ are consecutive integers, or $i=1$ and $j=h$; finally $\#\left(W_{i} \cap D\right)=1$ for every $i$.

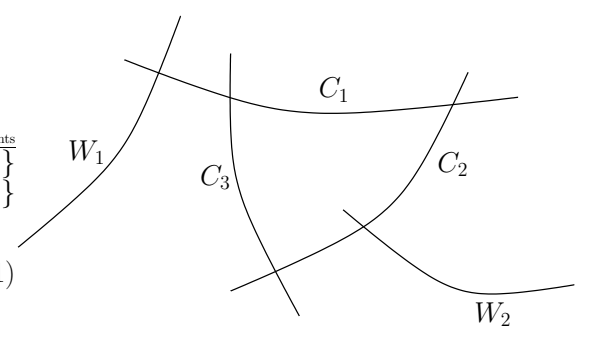

Case (2)

We shall see that the numerics of $S_{X}$ is the same in all cases.

In case (a) there are two even sets of nodes: $\emptyset$ and $\{N\}$. If $\Delta=\emptyset$ then $b_{1}(\Delta)=0$ and we find $2^{2 g-2}$ components of $S_{X}$, all having multiplicity 2 , supported on the quasistable curve obtained by "blowing up " all nodes of $X$.

If $\Delta=\{N\}$ then $b_{1}(\Delta)=1$ and we find $2^{2 g-1}$ reduced components of $S_{X}$ supported on the quasistable curve obtained by "blowing up " all nodes of $X$ but $N$.

In case (b) there are again two even sets of nodes: $\emptyset$ and $D_{\text {sing }}$ (the $h$ nodes of $D$ ). If $\Delta=\emptyset$ then $b_{1}(\Delta)=0$ and, just as before, there are $2^{2 g-2}$ components of $S_{X}$, all having multiplicity 2 .

If $\Delta=D_{\text {sing }}$ then $b_{1}(\Delta)=1$ and there are $2^{2 g-1}$ reduced components of $S_{X}$, supported on the quasistable curve obtained by "blowing up " all nodes of $X$, with the exception of the nodes of $D$.

The previous example is special in the sense that the numerical data of $S_{X}$ are independent of $X$ (so long as $b=1$ ). We leave it to the reader to check on other examples that for 
$b \geq 2$ this is no longer true. The picture will be made clear in the sequel (see Example 1 in the next section).

Example 3 (Split curves). A split curve $X$ is defined to be a stable curve made of two irreducible components, $X=C_{1} \cup C_{2}$, with $C_{i} \cong \mathbb{P}^{1}$; thus $C_{1}$ and $C_{2}$ meet in $g+1$ distinct points (the nodes of $X$ ), and $b=g$.

Clearly, a set $\Delta$ of nodes of $X$ is even if and only if its cardinality is even. Denote by $d=\# \Delta$, so that $b_{1}(\Delta)=d-1$, unless $d=0$ in which case $b_{1}(\emptyset)=0$.

Since $X$ has $\left(\begin{array}{c}g+1 \\ d\end{array}\right)$ even subsets of cardinality $d$, we see that, if $d$ is even and $0<d \leq g+1$, then $S_{X}$ possesses $\left(\begin{array}{c}g+1 \\ d\end{array}\right)$ irreducible components of multiplicity $2^{g-d+1}$; for $d=0, S_{X}$ has a unique component of multiplicity $2^{g}$.

Definition 6. Let $S$ be a scheme of pure dimension zero. Denote by $L(S)$ the multiplicity set of $S$, that is, set of integers occurring as multiplicities of components of $S$.

For example, $S$ is reduced if and only if $L(S)=\{1\}$.

We have, for a split curve $X$ of genus $g$,

$$
L\left(S_{X}\right)= \begin{cases}\left\{1,2^{2}, \ldots, 2^{g-3}, 2^{g-1}, 2^{g}\right\} & \text { if } g \text { is odd }, \\ \left\{2,2^{3}, \ldots, 2^{g-3}, 2^{g-1}, 2^{g}\right\} & \text { if } g \text { is even. }\end{cases}
$$

We have seen in Example 1 that curves of compact type are characterized by the fact that the multiplicity set of their scheme of spin curves is equal to $\{1\}$.

What is remarkable is that the analogue holds for split curves (Corollary 12). This is a consequence of a stronger result about the combinatorics of stable curves, to which the next section will be devoted.

\section{Graph theoretic results and applications}

\subsection{Preliminaries}

Let $\Gamma$ be a graph. We allow $\Gamma$ to have loops and multiple edges, namely: an edge can join a vertex to itself, and more than one edge can join two vertices (these kinds of graphs are sometimes called multigraphs in the literature). We also assume that $\Gamma$ has no isolated vertices.

We recall some basic definitions and properties, details can be found in Har69, Die91], for example.

We denote by $E(\Gamma)$ and $V(\Gamma)$ respectively the sets of edges and vertices of $\Gamma$. Let $\delta_{\Gamma}=\# E(\Gamma), \nu_{\Gamma}=\# V(\Gamma)$ and let $c(\Gamma)$ be the number of connected components of $\Gamma$. The first Betti number of $\Gamma$ is $b_{1}(\Gamma)=\delta_{\Gamma}-\nu_{\Gamma}+c(\Gamma)$. We shall say that a vertex (or an edge) $a$ is a separating vertex (a separating edge) if $c(\Gamma \backslash\{a\})>c(\Gamma)$.

A subgraph $\Gamma^{\prime} \subseteq \Gamma$ is a graph $\Gamma^{\prime}$ such that $V\left(\Gamma^{\prime}\right) \subseteq V(\Gamma)$ and $E\left(\Gamma^{\prime}\right) \subseteq E(\Gamma)$. Given two graphs $\Gamma_{1}, \Gamma_{2}$ their union is the graph $\Gamma_{1} \cup \Gamma_{2}$ such that $V\left(\Gamma_{1} \cup \Gamma_{2}\right)=V\left(\Gamma_{1}\right) \cup V\left(\Gamma_{2}\right)$ and $E\left(\Gamma_{1} \cup \Gamma_{2}\right)=E\left(\Gamma_{1}\right) \cup E\left(\Gamma_{2}\right)$.

The valency of a vertex is the number of edges ending in that vertex (a loop counting 2 in the valency). Clearly the sum of all valencies equals $2 \delta_{\Gamma}$. 
A circuit of $\Gamma$ is a subgraph of $\Gamma$ that has all valencies equal to 2 .

We consider a vector space associated to $\Gamma$, its cycle space $\mathcal{C}_{\Gamma}$. This is a vector space over $\mathbb{F}_{2}=\mathbb{Z} / 2 \mathbb{Z}$, of dimension $b_{1}(\Gamma)$. A 1-chain (respectively, 0-chain) of $\Gamma$ is a formal linear combination of edges (respectively, vertices) of $\Gamma$ with coefficients in $\mathbb{F}_{2}$.

Any 1-chain $\Delta=N_{1}+\cdots+N_{m}$ can be viewed as a subset $\left\{N_{1}, \ldots, N_{m}\right\}$ of $E(\Gamma)$, or as a subgraph of $\Gamma$, namely the smallest subgraph of $\Gamma$ containing $N_{1}, \ldots, N_{m}$. To avoid a heavy notation, we will not make any distinction among these different interpretations, denoting by the same letter, $\Delta$, the 1-chain, the set of edges and the induced graph.

The boundary operator $\partial$ sends 1 -chains to 0 -chains in the usual way: $\partial$ is linear and for an edge $N, \partial(N)=a+b$, where $a$ and $b$ are the vertices of $N$. The cycle space $\mathcal{C}_{\Gamma}$ is the kernel of the boundary operator (using the standard notation, $\mathcal{C}_{\Gamma}:=Z_{1}\left(\Gamma, \mathbb{F}_{2}\right)$ ). An element $\Delta \in \mathcal{C}_{\Gamma}$ is called a cyclic set or a cyclic subgraph of $\Gamma$.

Claim. A subgraph $\Delta \subseteq \Gamma$ is cyclic if and only if $\Delta$ has all even valencies.

Proof. Consider 1-chains and 0-chains with coefficients in $\mathbb{Z}$ instead of $\mathbb{F}_{2}$, and consider the 0 -chain $\partial(\Delta)$. The coefficient of any vertex in $\partial(\Delta)$ is exactly its valency in $\Delta$. Hence the statement follows.

In particular, a circuit is a cyclic set, and every cyclic set can be decomposed as an edge-disjoint union of circuits.

A graph $\Gamma$ having all even valencies, or equivalently such that $E(\Gamma) \in \mathcal{C}_{\Gamma}$, is called an eulerian graph. Such a graph is characterized by the existence of a closed walk passing exactly once through every edge.

Let now $X$ be a stable curve and $\Gamma=\Gamma_{X}$ be its dual graph. Let $\Delta \subset X_{\text {sing }}$ be a subset of nodes and (with the usual abuse of notation) $\Delta \subset \Gamma$ the corresponding subgraph.

Remark. To say that $\Delta$ is even in the sense of Definition 3 is exactly the same as saying that $\Delta \in \mathcal{C}_{\Gamma_{X}}$, or that $\Delta$ is cyclic, in the graph theoretic language. In particular, we see that on $X$ there are exactly $2^{b_{1}\left(\Gamma_{X}\right)}$ even subsets of nodes.

\subsection{Relating to spin curves}

As we saw in the previous section, the geometry of $S_{X}$ is ruled by the even sets of nodes of the stable curve $X$, that is, by the cyclic subgraphs of $\Gamma_{X}$.

Definition 7. The set of cyclic Betti numbers of a graph $\Gamma$ is $B_{\Gamma}:=\left\{b_{1}(\Delta) \mid \Delta \in \mathcal{C}_{\Gamma}\right\}$.

The relevance of such a definition comes from Proposition 5 . In fact, let $b=b_{1}\left(\Gamma_{X}\right)$, we have (see Definition 6)

$$
L\left(S_{X}\right)=\left\{2^{b-n} \mid n \in B_{\Gamma_{X}}\right\} .
$$

For example, the analysis of Example 11 shows that $X$ is of compact type if and only $B_{\Gamma_{X}}=\{0\}$. 
Elementary properties of $B_{\Gamma}$.

(P1) For all $m \in B_{\Gamma}$, we have $m \leq b_{1}(\Gamma)$.

In fact, for every subgraph $\Delta \subset \Gamma$, clearly $b_{1}(\Delta) \leq b_{1}(\Gamma)$.

(P2) $0 \in B_{\Gamma}$.

The vector space $\mathcal{C}_{\Gamma}$ contains the zero element.

(P3) $B_{\Gamma}=\{0\}$ if and only if $\Gamma$ is a tree, if and only if $1 \notin B_{\Gamma}$.

A circuit $\gamma$ always has $b_{1}(\gamma)=1$, and $b_{1}(\Gamma) \geq 1$ if and only if $\Gamma$ contains a circuit, if and only if $1 \in B_{\Gamma}$.

(P4) If $\Gamma^{\prime} \subset \Gamma$, then $\mathcal{C}_{\Gamma^{\prime}}$ is a subspace of $\mathcal{C}_{\Gamma}$ and $B_{\Gamma^{\prime}} \subseteq B_{\Gamma}$.

The vector space of 1-chains of $\Gamma^{\prime}$ is a subspace of the 1-chains of $\Gamma$.

(P5) If $\Gamma=\Gamma_{1} \cup \Gamma_{2}, E\left(\Gamma_{1}\right) \cap E\left(\Gamma_{2}\right)=\emptyset$ and $\# V\left(\Gamma_{1}\right) \cap V\left(\Gamma_{2}\right) \leq 1$, then $\mathcal{C}_{\Gamma}=\mathcal{C}_{\Gamma_{1}} \oplus \mathcal{C}_{\Gamma_{2}}$ and $B_{\Gamma}=B_{\Gamma_{1}}+B_{\Gamma_{2}}=\left\{n_{1}+n_{2} \mid n_{1} \in B_{\Gamma_{1}}, n_{2} \in B_{\Gamma_{2}}\right\}$.

Since $\Gamma=\Gamma_{1} \cup \Gamma_{2}$ and $E\left(\Gamma_{1}\right) \cap E\left(\Gamma_{2}\right)=\emptyset$, we have $\mathcal{C}_{\Gamma}=\mathcal{C}_{\Gamma_{1}} \oplus \mathcal{C}_{\Gamma_{2}}$. Since $\# V\left(\Gamma_{1}\right) \cap$ $V\left(\Gamma_{2}\right) \leq 1$, given two cyclic sets $\Delta_{1} \in \mathcal{C}_{\Gamma_{1}}$ and $\Delta_{2} \in \mathcal{C}_{\Gamma_{2}}$, either they are disjoint or they have one common vertex. In both cases we have $b_{1}\left(\Delta_{1} \cup \Delta_{2}\right)=b_{1}\left(\Delta_{1}\right)+b_{1}\left(\Delta_{2}\right)$.

(P6) A cyclic set does not contain any separating edge.

An edge $N$ is contained in some cyclic set if and only if it is not a separating edge: in fact, if $a$ and $b$ are the vertices of $N, N$ is not a separating edge if and only if there is a path in $\Gamma \backslash\{N\}$ joining $a$ and $b$. This path together with $N$ gives a cyclic set in $\Gamma$.

(P7) $b_{1}(\Gamma) \in B_{\Gamma}$ if and only if the set $E(\Gamma) \backslash\{$ separating edges $\}$ is a cyclic set.

Set $r=\#$ separating edges $\}$ and $\Gamma^{\prime}=\Gamma \backslash\{$ separating edges $\}$. Then we have $\delta_{\Gamma^{\prime}}=\delta_{\Gamma}-r, \nu_{\Gamma^{\prime}}=\nu_{\Gamma}$ and $c\left(\Gamma^{\prime}\right)=c(\Gamma)+r$, so $b_{1}\left(\Gamma^{\prime}\right)=b_{1}(\Gamma)$. Hence, if $\Gamma^{\prime}$ is a cyclic set, then $b_{1}(\Gamma) \in B_{\Gamma}$.

Suppose now that $\Delta$ is a cyclic set strictly smaller than $E(\Gamma) \backslash\{$ separating edges $\}$ : it suffices to show that $b_{1}(\Delta)<b_{1}(\Gamma)$. In fact, there exists a non-separating edge $N$ such that $\Delta$ is contained in the subgraph $\Gamma^{\prime}=\Gamma \backslash\{N\}$; we have $\delta_{\Gamma^{\prime}}=\delta_{\Gamma}-1, \nu_{\Gamma^{\prime}}=\nu_{\Gamma}$ and $c\left(\Gamma^{\prime}\right)=c(\Gamma)$, so $b_{1}(\Delta) \leq b_{1}\left(\Gamma^{\prime}\right)=b_{1}(\Gamma)-1$.

(P8) A graph $\Gamma$ is eulerian if and only if it has no separating edges and $b_{1}(\Gamma) \in B_{\Gamma}$.

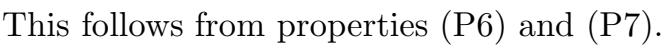

\section{Operations on $\Gamma$ that fix $\mathcal{C}_{\Gamma}$ and $B_{\Gamma}$ :}

1. elimination of a vertex of valency 1: we contract the unique edge containing the vertex;

2. elimination of a vertex of valency 2 , not allowed on the vertex of a loop: the two edges ending in the vertex are merged in a unique edge; 
3. elimination of a separating edge: the edge is contracted and gives rise to a separating vertex.

Lemma 8. If $\Gamma^{\prime}$ is obtained from $\Gamma$ by any sequence of operations of type 1,2 and 3, we have $b_{1}\left(\Gamma^{\prime}\right)=b_{1}(\Gamma), \mathcal{C}_{\Gamma^{\prime}} \simeq \mathcal{C}_{\Gamma}$ and $B_{\Gamma^{\prime}}=B_{\Gamma}$.

Proof. Elementary and easy.

Definition 9. A graph $\Gamma$ is superstable if all valencies of $\Gamma$ are at least 3, except possibly for the vertex of a loop.

For any graph $\Gamma$, there exists a unique superstable graph $\Gamma^{s}$ obtained from $\Gamma$ with a sequence of operations of type 1 and 2 , so that $\mathcal{C}_{\Gamma^{s}} \simeq \mathcal{C}_{\Gamma}$ and $B_{\Gamma^{s}}=B_{\Gamma}$.

To explain the choice of the name "superstable", pick a nodal connected curve $Z$, all of whose components have geometric genus zero and such that $\Gamma$ is the dual graph of $Z$. The fact that such a $Z$ may not be uniquely determined by $\Gamma$ is irrelevant in the following discussion. The point is that $\Gamma$ is superstable if and only if the curve $Z$ is stable (in the sense of Deligne and Mumford). Notice moreover that the three above operations on $\Gamma$ correspond to operations on $Z$ : the first contracts a smooth tail, the second contracts an exceptional component (in the sense of Definition 1), the third corresponds to smoothing a separating node. Hence operations 1 and 2 correspond to "stabilizing" operations on the curve; in other words, the graph $\Gamma^{s}$ defined above is the dual graph of the stable model of $Z$ (in the sense of Definition 11).

Example 4 (superstable graphs with $b_{1}(\Gamma)=1$ or 2 ). Let us only consider graphs free from separating edges, leaving it to the reader to list the remaining ones. It is immediate to see that if $b_{1}(\Gamma)=1, \Gamma$ must be a loop, and $B_{\Gamma}=\{0,1\}$ (this clarifies Example 2 in the previous section).

If $b_{1}(\Gamma)=2$, either $\Gamma$ has two loops as connected components, or it is connected and has all valencies at least 3 . In this last case, it is easy to see that the only possibilities for $\left(\delta_{\Gamma}, \nu_{\Gamma}\right)$ are $(2,1)$ and $(3,2)$, and that there is only one possible graph for each pair. We conclude that the superstable graphs with $b_{1}(\Gamma)=2$ and no separating edges are:

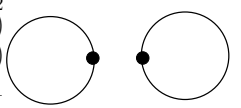

$B_{\Gamma}=\{0,1,2\}$

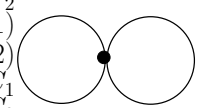

$B_{\Gamma}=\{0,1,2\}$

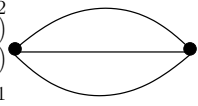

$B_{\Gamma}=\{0,1\}$

Now we introduce split graphs (compare with Example 3):

Definition 10. A graph is split if it is connected, has two vertices and no loops.

If $\Gamma$ is split and $b_{1}(\Gamma) \geq 2$, then $\Gamma$ is superstable.

Let $\Gamma$ be split and consider a subgraph $\Delta \subset \Gamma$ containing $r$ edges. Then $b_{1}(\Delta)=r-1$, and $\Delta$ is a cyclic set if and only if $r$ is even. Hence

$$
B_{\Gamma}=\left\{0, k \mid k \text { is odd and } k \leq b_{1}(\Gamma)\right\} .
$$




\subsection{Combinatorial results}

We now prove two structural characterizations of graphs, using their cyclic Betti numbers (Theorem 11 and Theorem 13).

Parenthetically, we mention that Theorem 11 and Corollary 12 generalize (and clarify) a crucial step in CS02 (Theorem 3.4.1); the main result of that paper is the fact that a general canonical curve is uniquely determined by the configuration of hyperplanes cutting theta characteristics on it. Applying stable reduction, such a classical, concrete problem is solved using the moduli theory of stable curves. The combinatorial analysis is used as a bridge between the projective and the abstract set-up.

Theorem 11. Let $\Gamma$ be a superstable graph such that $2 \notin B_{\Gamma}$. Then either $\Gamma$ is split, or $b_{1}(\Gamma)=1$ and $\Gamma$ is a loop, or $b_{1}(\Gamma)=3$ and $\Gamma$ is the tetrahedron:

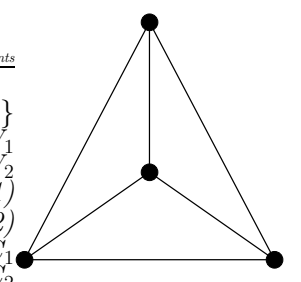

$$
B_{\Gamma}=\{0,1\}
$$

Proof. We remark first of all that $\Gamma$ is connected and does not have separating vertices. Otherwise, there would be two subgraphs $\Gamma_{1}, \Gamma_{2}$ such that $\Gamma=\Gamma_{1} \cup \Gamma_{2}, E\left(\Gamma_{1}\right) \cap E\left(\Gamma_{2}\right)=\emptyset$ and $\# V\left(\Gamma_{1}\right) \cap V\left(\Gamma_{2}\right) \leq 1$. Since $\Gamma$ is superstable, $\Gamma_{i}$ cannot be a tree, for $i=1,2$. By

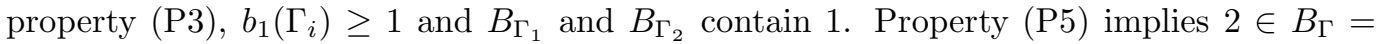
$B_{\Gamma_{1}}+B_{\Gamma_{2}}$, a contradiction.

From the fact that $\Gamma$ has no separating vertices, we deduce that $\Gamma$ has no separating edges. If it did, let $\Gamma^{\prime}$ be the superstable graph obtained by contracting all separating edges; since every such contraction generates a separating vertex, $\Gamma^{\prime}$ would possess separating vertices. On the other hand, by Lemma 8, $B_{\Gamma}=B_{\Gamma^{\prime}}$, therefore $\Gamma^{\prime}$ satisfies the Theorem's assumptions, hence, by what we proved above, $\Gamma^{\prime}$ has no separating vertices; a contradiction.

We proceed by induction on $b_{1}(\Gamma)$. If $b_{1}(\Gamma)=1$ or 2 , the statement follows from Example 1 .

Let's suppose $b_{1}(\Gamma) \geq 3$. We choose an edge $N \in E(\Gamma)$ and consider the subgraph $\widetilde{\Gamma}=\Gamma \backslash\{N\}$. $\widetilde{\Gamma}$ could have one or two vertices of valency 2 ; we eliminate those vertices (operation 2) and obtain a connected, superstable graph $\Gamma^{\prime}$ such that $b_{1}\left(\Gamma^{\prime}\right)=b_{1}(\widetilde{\Gamma})=$ $b_{1}(\Gamma)-1$. By Lemma 8 and property $(\mathrm{P} \rrbracket)$ we have $B_{\Gamma^{\prime}}=B_{\widetilde{\Gamma}} \subseteq B_{\Gamma}$, so $2 \notin B_{\Gamma^{\prime}}$ : the induction hypothesis implies that either $\Gamma^{\prime}$ is split, or $b_{1}(\Gamma)=4$ and $\Gamma^{\prime}$ is the tetrahedron.

Suppose first that $\Gamma^{\prime}$ is split. If $\Gamma$ is not split, there are three possibilities for $\Gamma$ :
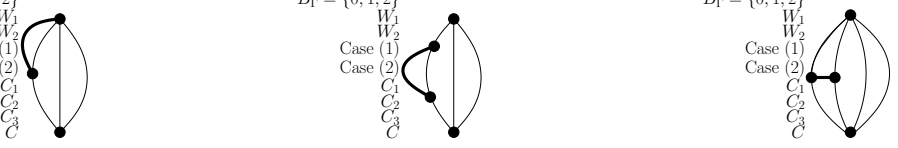
The thickened edge in the picture is $N$. Recall that $b_{1}\left(\Gamma^{\prime}\right) \geq 2$.

In the first two cases we obtain a contradiction because there clearly is a cyclic set $\Delta \subset \Gamma$ such that $b_{1}(\Delta)=2$ :
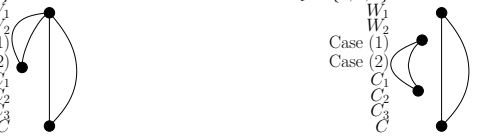

In the third case, if $b_{1}\left(\Gamma^{\prime}\right) \geq 3$, we can find again a cyclic set $\Delta \subset \Gamma$ with $b_{1}(\Delta)=2$ (picture below, on the left), while if $b_{1}\left(\Gamma^{\prime}\right)=2$ we get the tetrahedron (picture below, on the right):
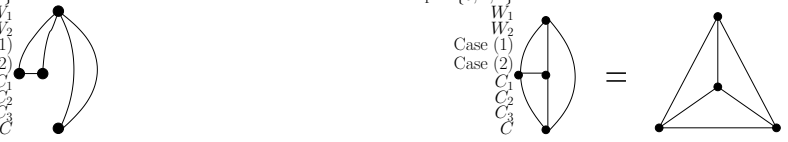

Suppose now that $\Gamma^{\prime}$ is the tetrahedron: then in any case we get a cyclic set $\Delta \subset \Gamma$ such that $b_{1}(\Delta)=2$ :

$\Gamma:$
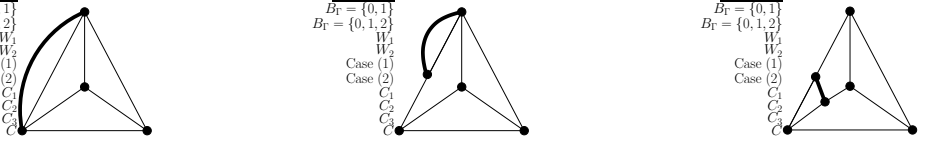

$\Delta:$
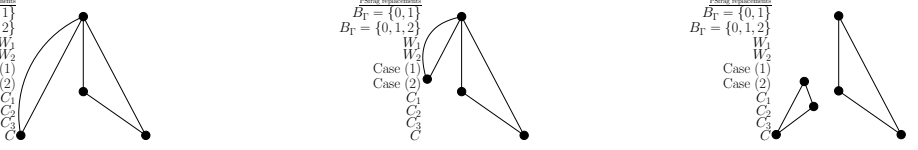

Applying this to stable curves we obtain:

Corollary 12. Let $X$ be a stable curve of genus g. Suppose that $S_{X}$ has a component of multiplicity $2^{g}$ and no component of multiplicity $2^{g-2}$. Then either $X$ is a split curve or $X$ has genus 3 and it is the polygonal curve.

(Recall that the polygonal curve of genus 3 is the one whose dual graph is a tetrahedron.)

Proof. The fact that $2^{g} \in S_{X}$ implies, by Proposition 5, that $b_{1}\left(\Gamma_{X}\right)=g$ and hence every irreducible component of $X$ has geometric genus 0 . Since $X$ is stable, $\Gamma_{X}$ must have all valencies at least equal to 3 . To say that $2^{g-2} \notin S_{X}$ is the same as saying that $2 \notin B_{\Gamma_{X}}$. Therefore all the assumptions of Theorem 11 are satisfied and $X$ is a split curve or its dual graph is the tetrahedron.

Theorem 13. Let $\Gamma$ be a superstable graph. Suppose that $3 \notin B_{\Gamma}$ and that there exists $m \in B_{\Gamma}$ such that $m>3$. Then $b_{1}(\Gamma)=4$ and $\Gamma$ is the "fat-triangle": 


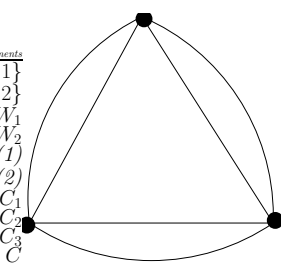

Proof. Remark that $b_{1}(\Gamma) \geq m \geq 4$, so by Theorem 11 we know that $2 \in B_{\Gamma}$. Moreover, $\Gamma$ is connected and does not have separating vertices. Otherwise, there would be two subgraphs $\Gamma_{1}, \Gamma_{2}$ such that $\Gamma=\Gamma_{1} \cup \Gamma_{2}, E\left(\Gamma_{1}\right) \cap E\left(\Gamma_{2}\right)=\emptyset$ and $\# V\left(\Gamma_{1}\right) \cap V\left(\Gamma_{2}\right) \leq 1$. Since $\Gamma$ is

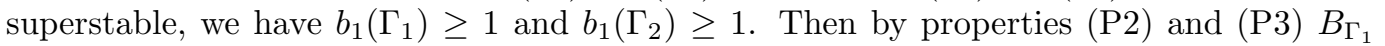
and $B_{\Gamma_{2}}$ contain 0 and 1 , but since $B_{\Gamma}=B_{\Gamma_{1}}+B_{\Gamma_{2}}$ and $3 \notin B_{\Gamma}$, we must have that $2,3 \notin B_{\Gamma_{i}}$ for $i=1,2$. We deduce from Theorem 11 that $B_{\Gamma_{i}}=\{0,1\}$, so $B_{\Gamma}=\{0,1,2\}$, a contradiction.

Just as we did in the proof of Theorem 11, we deduce that $\Gamma$ has no separating edges.

We continue by induction on $b_{1}(\Gamma)$. When $b_{1}(\Gamma)=4$, we have $B_{\Gamma}=\{0,1,2,4\}$. Since $4=b_{1}(\Gamma) \in B_{\Gamma}, \Gamma$ is eulerian (property $(\mathrm{P} 8)$ ); in particular all valencies are at least 4. Thus the possibilities for the number of edges and vertices $\left(\delta_{\Gamma}, \nu_{\Gamma}\right)$ are only $(4,1),(5,2)$ and $(6,3)$. Now, the only possible $\Gamma$ having $b_{1}(\Gamma)=4$, even valencies and without separating vertices is the fat-triangle.

Suppose now $b_{1}(\Gamma)>4$.

Claim. There exists an $m \in B_{\Gamma}$ such that $3<m<b_{1}(\Gamma)$.

Let's see how the claim implies the statement. Let $\Delta \subset \Gamma$ be a cyclic set such that $b_{1}(\Delta)=m$. Since $m<b_{1}(\Gamma)$, there exists an edge $N \in E(\Gamma) \backslash E(\Delta)$. Consider the superstable graph $\Gamma^{\prime}$ obtained from $\Gamma$ eliminating $N$, as in the proof of Theorem 11. Then $B_{\Gamma^{\prime}} \subseteq B_{\Gamma}$, so $3 \notin B\left(\Gamma^{\prime}\right)$, but clearly $\Delta \subseteq \Gamma^{\prime}$, so $m \in B\left(\Gamma^{\prime}\right)$. Since $b_{1}\left(\Gamma^{\prime}\right)=b_{1}(\Gamma)-1$, by the induction hypothesis we deduce that $b_{1}(\Gamma)=5$ and $\Gamma^{\prime}$ is the fat-triangle. Then there are five possibilities for $\Gamma$ :
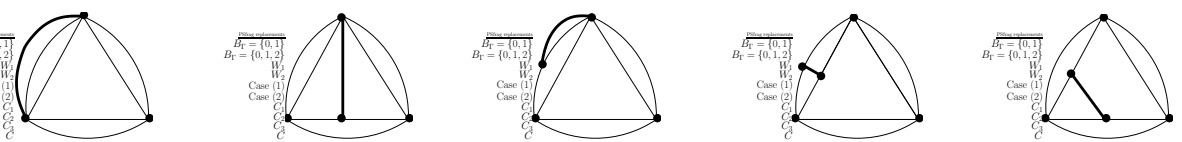

The thickened edge in the picture is $N$. In all these cases, there is a cyclic set $\Delta$ with $b_{1}(\Delta)=3$, against the hypotheses:
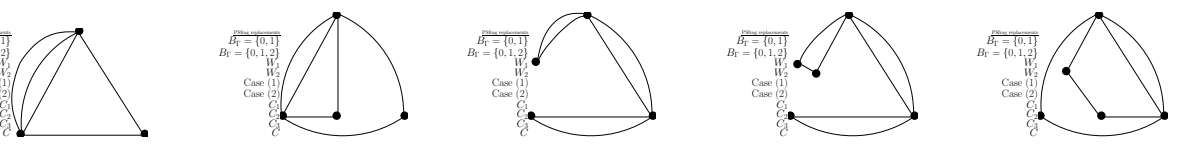

Proof of the claim. By contradiction, suppose that $B_{\Gamma}=\left\{0,1,2, b_{1}(\Gamma)\right\}$. Since $b_{1}(\Gamma) \in B_{\Gamma}$, $\Gamma$ is eulerian by property (PB). In particular, we can decompose $\Gamma$ as an edge-disjoint union of circuits: namely, $\Gamma=\gamma_{1} \cup \cdots \cup \gamma_{r}$ and $E\left(\gamma_{i}\right) \cap E\left(\gamma_{j}\right)=\emptyset$ for all $i, j$. Since there are only a 
finite number of possibilities for such a decomposition of $\Gamma$, we can choose a decomposition with $r$ maximal.

For all $i, j=1, \ldots, r$, we have $\# V\left(\gamma_{i}\right) \cap V\left(\gamma_{j}\right) \leq 1$ and $b_{1}\left(\gamma_{i} \cup \gamma_{j}\right) \leq 2$. In fact, if $\gamma_{i}$ and $\gamma_{j}$ have $s \geq 3$ common vertices, then $\gamma_{i} \cup \gamma_{j}$ has a decomposition as an edge-disjoint union of $s$ circuits, hence we get a decomposition of $\Gamma$ in $r+s-2$ circuits, against the maximality of $r$. Moreover, we have

$$
\begin{aligned}
b_{1}\left(\gamma_{i} \cup \gamma_{j}\right) & =\delta_{\gamma_{i} \cup \gamma_{j}}-\nu_{\gamma_{i} \cup \gamma_{j}}+c\left(\gamma_{i} \cup \gamma_{j}\right) \\
& =\delta_{\gamma_{i}}+\delta_{\gamma_{j}}-\nu_{\gamma_{i}}-\nu_{\gamma_{j}}+\# V\left(\gamma_{i}\right) \cap V\left(\gamma_{j}\right)+c\left(\gamma_{i} \cup \gamma_{j}\right) \\
& =\# V\left(\gamma_{i}\right) \cap V\left(\gamma_{j}\right)+c\left(\gamma_{i} \cup \gamma_{j}\right) .
\end{aligned}
$$

If $\# V\left(\gamma_{i}\right) \cap V\left(\gamma_{j}\right)=2$, then $b_{1}\left(\gamma_{i} \cup \gamma_{j}\right)=3 \in B_{\Gamma}$, a contradiction: so $\# V\left(\gamma_{i}\right) \cap V\left(\gamma_{j}\right) \leq 1$ and $b_{1}\left(\gamma_{i} \cup \gamma_{j}\right) \leq 2$.

Consider now the cyclic set $\gamma_{1} \cup \cdots \cup \gamma_{r-1}$ : since $b_{1}\left(\gamma_{1} \cup \cdots \cup \gamma_{r-1}\right) \in B_{\Gamma}$, we get

$$
r-1 \leq b_{1}\left(\gamma_{1} \cup \cdots \cup \gamma_{r-1}\right) \leq 2,
$$

thus $r \leq 3$. Since $r \leq 2$ would imply $b_{1}(\Gamma) \leq 3$, we obtain $r=3$. Then we have

$$
\begin{aligned}
5 & \leq b_{1}(\Gamma)=\delta_{\Gamma}-\nu_{\Gamma}+1 \\
& =\sum_{i=1}^{3} \delta_{\gamma_{i}}-\sum_{i=1}^{3} \nu_{\gamma_{i}}+\sum_{1 \leq i<j \leq 3} \# V\left(\gamma_{i}\right) \cap V\left(\gamma_{j}\right)-\# V\left(\gamma_{1}\right) \cap V\left(\gamma_{2}\right) \cap V\left(\gamma_{3}\right)+1 \\
& \leq \sum_{1 \leq i<j \leq 3} \# V\left(\gamma_{i}\right) \cap V\left(\gamma_{j}\right)+1 \leq 4,
\end{aligned}
$$

a contradiction.

We conclude with a simple consequence involving stable spin curves

Corollary 14. Let $X$ be a stable curve of genus at least 4, having superstable dual graph.

(i) If $2^{b_{1}\left(\Gamma_{X}\right)-2} \notin L\left(S_{X}\right)$, then $X$ is the union of two smooth, irreducible components meeting in $b_{1}\left(\Gamma_{X}\right)+1$ points.

(ii) If $2^{b_{1}\left(\Gamma_{X}\right)-3} \notin L\left(S_{X}\right)$, and there exists $m \in L\left(S_{X}\right)$ such that $m<2^{b_{1}\left(\Gamma_{X}\right)-3}$, then $b_{1}\left(\Gamma_{X}\right)=4$ and the dual graph of $X$ is a fat triangle.

Where the "fat triangle" is defined in Theorem 13.

Proof. Just observe that $(i)$ and (ii) translate, respectively, Theorem 11 and Theorem 13. 


\section{References}

[AK90] Allen B. Altman and Steven L. Kleiman. The presentation functor and the compactified Jacobian. In The Grothendieck Festschrift, Volume I, volume 86 of Progress in Mathematics, pages 15-32. Birkhäuser, 1990.

[Cap94] Lucia Caporaso. A compactification of the universal Picard variety over the moduli space of stable curves. Journal of the American Mathematical Society, 7:589-660, 1994.

[Cor89] Maurizio Cornalba. Moduli of curves and theta-characteristics. In Lectures on Riemann Surfaces: Proceedings of the College on Riemann Surfaces, International Centre for Theoretical Physics, Trieste, 1987, pages 560-589. World Scientific, 1989.

[CS02] Lucia Caporaso and Edoardo Sernesi. Characterizing curves by their odd theta-characteristics. Preprint $\backslash$ protect \vrule width0pt \protect \href\{http://arxiv.org/abs/math/0204164\}\{math.AG/0204164\}, 2002.

[Die91] Reinhard Diestel. Graph Theory, volume 173 of Graduate Texts in Mathematics. Springer, 1991.

[DM69] Pierre Deligne and David Mumford. The irreducibility of the space of curves of given genus. Publications Mathématiques de l'Institut des Hautes Études Scientifiques, 36:75109, 1969.

[EGK02] Eduardo Esteves, Mathieu Gagné, and Steven L. Kleiman. Autoduality of the compactified Jacobian. Journal of the London Mathematical Society, 65(3):591-610, 2002.

[Fon02] Claudio Fontanari. On the geometry of the compactification of the universal Picard variety. Preprint $\backslash$ protect \vrule width0pt \protect \href \{http://arxiv.org/abs/math/0202168\} \{math. AG/0202168\}, 2002.

[Har69] Frank Harary. Graph Theory. Addison-Wesley Publishing Company, 1969.

[Kle84] Steven L. Kleiman. The structure of the compactified Jacobian: a review and announcement. In Seminari di Geometria 1982-1983. Università degli Studi di Bologna, Dipartimento di Matematica, pages 81-92. 1984. 